\title{
Sobolev inequalities for probability measures on the real line
}

\author{
by \\ F. BARthe and C. Roberto (Marne-la-Vallée)
}

\begin{abstract}
We give a characterization of those probability measures on the real line which satisfy certain Sobolev inequalities. Our starting point is a simpler approach to the Bobkov-Götze characterization of measures satisfying a logarithmic Sobolev inequality. As an application of the criterion we present a soft proof of the Latała-Oleszkiewicz inequality for exponential measures, and describe the measures on the line which have the same property. New concentration inequalities for product measures follow.
\end{abstract}

1. Introduction. Poincaré and logarithmic Sobolev inequalities are essential tools in the study of concentration of measure and in the estimation of the relaxation time of various ergodic systems (see e.g. [12, 1, 8, 17, 10]). Recall that a probability measure $\mu$ on $\mathbb{R}^{n}$ satisfies a Poincaré (or spectral gap) inequality if there exists a constant $C_{\mathrm{P}}>0$ such that every smooth function $f: \mathbb{R}^{n} \rightarrow \mathbb{R}$ satisfies

$$
\int f^{2} d \mu-\left(\int f d \mu\right)^{2} \leq C_{\mathrm{P}} \int|\nabla f|^{2} d \mu .
$$

Here $|\cdot|$ is the Euclidean norm. One could also consider more general Dirichlet forms than $|\nabla f|^{2}$. On the other hand, $\mu$ satisfies a logarithmic Sobolev inequality if there exists a constant $C_{\mathrm{LS}}>0$ so that for every smooth $f$ one has

$$
\operatorname{Ent}_{\mu}\left(f^{2}\right) \leq C_{\mathrm{LS}} \int|\nabla f|^{2} d \mu
$$

where the entropy is defined by

$$
\operatorname{Ent}_{\mu}\left(f^{2}\right)=\int f^{2} \log f^{2} d \mu-\left(\int f^{2} d \mu\right) \log \left(\int f^{2} d \mu\right) .
$$

This property was introduced by Gross [7] and is stronger than the Poincaré inequality. For example the standard Gaussian measure, say on $\mathbb{R}$, satisfies a Poincaré and a logarithmic Sobolev inequality whereas the double expo-

2000 Mathematics Subject Classification: 26D10, 60E15.

Key words and phrases: Sobolev inequalities, concentration. 
nential measure only satisfies a spectral gap inequality. Beckner [3] showed that Gaussian measures satisfy a family of Sobolev inequalities interpolating between (1) and (2). More recently, Latała and Oleszkiewicz [11] were able to establish a corresponding fact for the probability measures $\nu_{r}, r \in(1,2)$, defined by

$$
d \nu_{r}(t)=\frac{e^{-|t|^{r}} d t}{2 \Gamma(1+1 / r)}, \quad t \in \mathbb{R} .
$$

Namely, there is a universal constant $C>0$ such that for every smooth $f: \mathbb{R} \rightarrow \mathbb{R}$ and every $p \in(1,2)$, one has

$$
\int f^{2} d \nu_{r}-\left(\int|f|^{p} d \nu_{r}\right)^{2 / p} \leq C(2-p)^{2(1-1 / r)} \int f^{\prime 2} d \nu_{r}
$$

For $r=2$ and $C=1$ this is Beckner's interpolated inequality. The proof of Latała and Oleszkiewicz is hard, and the inequality itself is quite subtle: most of the information is encoded in the speed at which the constant vanishes as $p$ tends to 2 . Their result nicely completed the picture: while a Poincaré inequality ensures exponential concentration, and a log-Sobolev inequality yields Gaussian concentration, the family of Sobolev inequalities (3) with constant $(2-p)^{2(1-1 / r)}$ ensures concentration with decay $\exp \left(-K t^{r}\right), t \geq 1$, for the underlying measure. Moreover, like the Poincaré and the logarithmic Sobolev inequalities, the interpolated inequalities also have the tensorization property [11]. For this very reason it is of interest to study them for measures on the real line, since this automatically provides information on their infinite products.

The original motivation of this work was to characterize the probability measures on $\mathbb{R}$ which satisfy the same inequalities (3) as the measure $\nu_{r}$, for any $r \in(1,2)$. As we explain later, this was already done for log-Sobolev and Poincaré inequalities (which correspond to $r=2$ and $r=1$ ), by means of Hardy type inequalities. Before giving some background about Hardy inequalities, we would like to emphasize that all the previous inequalities enter the natural framework of Sobolev inequalities of the form

$$
\frac{\left(\int|f|^{p} d \mu\right)^{2 / p}-\int f^{2} d \mu}{p-2} \leq C \int|\nabla f|^{2} d \mu .
$$

Indeed, Poincaré corresponds to $p=1$ and log-Sobolev to the limit case when $p$ tends to 2 . The latter framework also encompasses more classical Sobolev inequalities, like the spherical one, for $p>2$.

The Hardy inequality was originally introduced by Hardy, Littlewood and Pólya $[9 ; 21$, p. 20]. They proved, for $p \in[1, \infty)$ and $b \in \mathbb{R}$ with $b p<-1$, that 


$$
\left(\sum_{x=0}^{\infty} x^{b}\left|\sum_{y=0}^{x} f(y)\right|^{p}\right)^{1 / p} \leq \frac{-p}{b p+1}\left(\sum_{x=0}^{\infty}\left|x^{b+1} f(x)\right|^{p}\right)^{1 / p}
$$

for every function $f$ on $\mathbb{N}$ (the constant is optimal). Tomaselli [20] and Talenti [19] extended the inequality to general weight functions instead of $x^{b}$ and $x^{b+1}$ and also to the continuous setting (the weights being absolutely continuous measures). Let us specify that all the measures considered in our article are non-negative. Muckenhoupt [15] established the following statement for general measures. Let $\mu, \nu$ be Borel measures on $\mathbb{R}^{+}$and let $p>1$. Then the best constant $A$ so that every smooth function $f$ satisfies

$$
\left(\int_{0}^{\infty}(f(x)-f(0))^{p} d \mu(x)\right)^{1 / p} \leq A\left(\int_{0}^{\infty} f^{\prime p} d \nu\right)^{1 / p}
$$

is finite if and only if

$$
B=\sup _{x>0} \mu([x, \infty))^{1 / p}\left(\int_{0}^{x} \frac{1}{n(t)^{p^{\prime} / p}} d t\right)^{1 / p^{\prime}}
$$

is finite (throughout the paper we adopt the convention that $0 \cdot \infty=0$, in other words the supremum is only over $x \in(0, \max (\operatorname{supp} \mu)])$. Here $p^{\prime}$ is defined by $1 / p+1 / p^{\prime}=1$ and $n$ stands for the density of the absolutely continuous part of $\nu$. Moreover, when $A$ is finite, $B \leq A \leq p^{1 / p} p^{1 / p^{\prime}} B$.

Using Hardy inequalities via the latter result by Muckenhoupt, Bobkov and Götze [4] gave the following characterization of those probability measures on $\mathbb{R}$ which satisfy a logarithmic Sobolev inequality (2):

ThEOREM $1([4])$. Let $\mu, \nu$ be Borel measures on $\mathbb{R}$ with $\mu(\mathbb{R})=1$, and let $n(t) d t$ denote the absolutely continuous component of $\nu$. Let $m$ be a median of $\mu$. Let $C$ be the optimal constant such that for every smooth $f: \mathbb{R} \rightarrow \mathbb{R}$ one has

$$
\operatorname{Ent}_{\mu}\left(f^{2}\right) \leq C \int f^{\prime 2} d \nu
$$

Then $\frac{1}{150}\left(D_{0}+D_{1}\right) \leq C \leq 468\left(D_{0}+D_{1}\right)$ where

$$
\begin{aligned}
& D_{0}=\sup _{x<m} \mu((-\infty, x]) \log \left(\frac{1}{\mu((-\infty, x])}\right) \int_{x}^{m} \frac{1}{n}, \\
& D_{1}=\sup _{x>m} \mu([x, \infty)) \log \left(\frac{1}{\mu([x, \infty))}\right) \int_{m}^{x} \frac{1}{n} .
\end{aligned}
$$

Apparently, this was the first time that Hardy inequalities had been used in probability theory. Next Miclo [14] and Chen [5, 6] extended their approach to Poincaré and more general inequalities, including Sobolev in- 
equalities (see also [1]). We wish to emphasize here that one can derive from these results a simple expression for the best constant $C$ in a Sobolev inequality like (4), up to constants depending on $p$. However, these constants degenerate when $p$ tends to 2 , so these results are not precise enough to address the inequalities of Latała and Oleszkiewicz.

The rest of the paper is divided into three sections. In Section 2 we present a simpler and tighter proof for the latter theorem of Bobkov and Götze on log-Sobolev inequalities. The keystone in their approach was to replace the entropy $\operatorname{Ent}\left(f^{2}\right)$ by some Orlicz norm $\left\|f^{2}\right\|_{\psi}$, for which classical Banach space theory provides a representation of the form $\left\|f^{2}\right\|_{\psi}=$ $\sup _{g \in \mathcal{G}} \int f^{2} g d \mu$, where $\mathcal{G}$ is a set of non-negative functions. This representation allows one to reduce the log-Sobolev inequality to a family of Hardy inequalities. Instead, we give a new class $\mathcal{G}$ that naturally controls the entropy.

Section 3 is devoted to the extension of our method to Sobolev inequalities like (4) for measures on the line and $p \in(1,2)$, and to the inequalities of Latała and Oleszkiewicz. We manage to characterize those probability measures on the line which satisfy their inequalities. This in turn provides a soft proof of their result for $\nu_{r}$, and gives new concentration inequalities for product measures.

For the sake of clarity, the arguments of Sections 2 and 3 are not written in full generality. Section 4 collects remarks on possible extensions, for example to discrete settings, or to measures on $\mathbb{R}^{n}$. In this situation we introduce the right notion of capacity of a set with respect to a probability measure.

2. Logarithmic Sobolev inequalities. Our starting point is the following statement, taken from [4], which is a direct corollary of the previously quoted criterion of Muckenhoupt. Let $\mu, \nu$ be (non-negative) Borel measures on $[m, \infty)$ and let $n(x) d x$ be the absolutely continuous component of $\nu$. Let $G$ be a family of non-negative Borel measurable functions on $[m, \infty)$, and for any measurable function $f$ set

$$
\Phi(f)=\sup _{g \in G} \int_{m}^{\infty} f g d \mu .
$$

With this notation one has

Proposition 2 ([4]). Let $A$ be the smallest constant such that for every smooth function $f$ with $f(m)=0$ one has

$$
\Phi\left(f^{2}\right) \leq A \int_{m}^{\infty} f^{\prime 2} d \nu .
$$


Then $B \leq A \leq 4 B$ where

$$
B=\sup _{x>m} \Phi\left(\mathbf{1}_{[x, \infty)}\right) \int_{m}^{x} \frac{d t}{n(t)} .
$$

This result seems adapted to the study of logarithmic Sobolev inequalities, due to the classical variational expression for the entropy of a realvalued non-negative measurable function $\varphi$ on a probability space $(X, P)$ :

$$
\operatorname{Ent}_{P}(\varphi)=\sup \left\{\int_{X} \varphi g d P ; \int_{X} e^{g} d P \leq 1\right\} .
$$

However one should note that the only non-negative function $g$ in the latter supremum is the zero function, so this representation is not of the form required in the proposition. We show in the rest of this section that a little more work allows us to overcome this difficulty. We obtain the following refinement of the criterion of Bobkov and Götze:

TheORem 3. Let $\mu, \nu$ be Borel measures on $\mathbb{R}$ with $\mu(\mathbb{R})=1$ and $d \nu(x)$ $=n(x) d x$. Let $m$ be a median of $\mu$. Let $C$ be the optimal constant such that for every smooth $f: \mathbb{R} \rightarrow \mathbb{R}$ one has

$$
\operatorname{Ent}_{\mu}\left(f^{2}\right) \leq C \int f^{\prime 2} d \nu .
$$

Then $\max \left(b_{-}, b_{+}\right) \leq C \leq 4 \max \left(B_{-}, B_{+}\right)$where

$$
\begin{aligned}
& b_{+}=\sup _{x>m} \mu([x, \infty)) \log \left(1+\frac{1}{2 \mu([x, \infty))}\right) \int_{m}^{x} \frac{1}{n}, \\
& B_{+}=\sup _{x>m} \mu([x, \infty)) \log \left(1+\frac{e^{2}}{\mu([x, \infty))}\right) \int_{m}^{x} \frac{1}{n} \\
& b_{-}=\sup _{x<m} \mu((-\infty, x]) \log \left(1+\frac{1}{2 \mu((-\infty, x])}\right) \int_{x}^{m} \frac{1}{n}, \\
& B_{-}=\sup _{x<m} \mu((-\infty, x]) \log \left(1+\frac{e^{2}}{\mu((-\infty, x])}\right) \int_{x}^{m} \frac{1}{n} .
\end{aligned}
$$

We shall use the following lemmas. The first one is due to Rothaus, it appears in previous proofs, and allows us to restrict to functions that vanish at a given point.

Lemma 4 ([16]). On a probability space $(X, P)$, let $f: X \rightarrow \mathbb{R}$ with $\int_{X} f^{2} d P<\infty$. Then for every $a \in \mathbb{R}$ one has

$$
\operatorname{Ent}_{P}\left(f^{2}\right) \leq \operatorname{Ent}_{P}\left((f-a)^{2}\right)+2 \int(f-a)^{2} d P .
$$


The next lemmas, though rather simple, are crucial to our argument.

LEMMA 5. Let $\varphi$ be a non-negative measurable function on a probability space $(X, P)$. Then

$$
\operatorname{Ent}_{P}(\varphi)+2 \int_{X} \varphi d P \leq \sup \left\{\int_{X} \varphi h d P ; \int_{X} e^{h} d P \leq e^{2}+1 \text { and } h \geq 0\right\} .
$$

Proof. By the variational characterization of entropy (6), and setting $h=g+2$, one has

$$
\begin{aligned}
\operatorname{Ent}_{P}(\varphi)+2 \int_{X} \varphi & =\sup \left\{\int_{X}(g+2) \varphi d P ; \int_{X} e^{g} d P \leq 1\right\} \\
& =\sup \left\{\int_{X} h \varphi d P ; \int_{X} e^{h} d P \leq e^{2}\right\} \\
& \leq \sup \left\{\int_{X} \varphi h \mathbf{1}_{h \geq 0} d P ; \int_{X} e^{h} d P \leq e^{2}\right\} .
\end{aligned}
$$

Since $\int_{X} e^{h \mathbf{1}_{h \geq 0}} d P=\int_{X} e^{h} \mathbf{1}_{h \geq 0} d P+\int_{X} \mathbf{1}_{h<0} d P \leq e^{2}+1$, the result follows.

Lemma 6. Let $Q$ be a finite measure on a space $X$. Let $K>Q(X)$ and let $A \subset X$ be measurable with $Q(A)>0$. Then

$$
\sup \left\{\int_{X} \mathbf{1}_{A} h d Q ; \int_{X} e^{h} d Q \leq K \text { and } h \geq 0\right\}=Q(A) \log \left(1+\frac{K-Q(X)}{Q(A)}\right) .
$$

Proof. Write $S$ for the above supremum. In $\int_{A} h d Q$, the values of $h$ on the complement of $A$ do not matter. So the best choice is to take $h$ minimal (that is, $h=0$ ) on $A^{\mathrm{c}}$, in order to save on the constraint $\int e^{h} d Q \leq K$. This shows that

$$
S=\sup \left\{\int_{A} h d Q ; Q\left(A^{\mathrm{c}}\right)+\int_{A} e^{h} d Q \leq K \text { and } h \geq 0\right\} .
$$

Concavity of the logarithm ensures that

$$
\int_{A} h \frac{d Q}{Q(A)} \leq \log \int_{A} e^{h} \frac{d Q}{Q(A)}
$$

which readily implies that $S \leq Q(A) \log \left(\left(K-Q\left(A^{\mathrm{c}}\right)\right) / Q(A)\right)$. There is actually equality as one can check with

$$
h=\mathbf{1}_{A} \log \left(1+\frac{K-Q(X)}{Q(A)}\right) \geq 0
$$

Proof of Theorem 3. We start with the upper bound on the best $C$ in the logarithmic Sobolev inequality. Let $f: \mathbb{R} \rightarrow \mathbb{R}$ be smooth. We consider 
the functions $F=f-f(m), F_{+}=F \mathbf{1}_{(m, \infty)}$ and $F_{-}=F \mathbf{1}_{(-\infty, m)}$. They are continuous. Since $F^{2}=F_{+}^{2}+F_{-}^{2}$ one has

$$
\begin{aligned}
\operatorname{Ent}_{\mu}\left(F^{2}\right) & =\sup \left\{\int F_{+}^{2} g d \mu+\int F_{-}^{2} g d \mu ; \int e^{g} d \mu \leq 1\right\} \\
& \leq \operatorname{Ent}_{\mu}\left(F_{+}^{2}\right)+\operatorname{Ent}_{\mu}\left(F_{-}^{2}\right),
\end{aligned}
$$

where we have used the fact that the supremum of a sum is less than the sum of the suprema. By Lemma 4 and the previous remarks, one gets

$$
\begin{aligned}
\operatorname{Ent}_{\mu}\left(f^{2}\right) & \leq \operatorname{Ent}_{\mu}\left(F^{2}\right)+2 \int F^{2} d \mu \\
& \leq \operatorname{Ent}_{\mu}\left(F_{+}^{2}\right)+2 \int F_{+}^{2} d \mu+\operatorname{Ent}_{\mu}\left(F_{-}^{2}\right)+2 \int F_{-}^{2} d \mu .
\end{aligned}
$$

Next we work separately with $F_{+}$. Recall that it is identically zero on $(-\infty, m]$. By Lemma 5 ,

$$
\operatorname{Ent}_{\mu}\left(F_{+}^{2}\right)+2 \int F_{+}^{2} d \mu \leq \sup \left\{\int F_{+}^{2} h d \mu ; \int e^{h} d \mu \leq e^{2}+1 \text { and } h \geq 0\right\} .
$$

Applying Proposition 2, we get

$$
\operatorname{Ent}_{\mu}\left(F_{+}^{2}\right)+2 \int F_{+}^{2} d \mu \leq 4 \widetilde{B}_{+} \int\left(F_{+}^{\prime}\right)^{2} d \nu
$$

where

$$
\widetilde{B}_{+}=\sup _{x>m}\left[\sup \left\{\int \mathbf{1}_{[x, \infty)} h d \mu ; \int e^{h} d \mu \leq e^{2}+1 \text { and } h \geq 0\right\} \int_{m}^{x} \frac{1}{n}\right] .
$$

Lemma 6 ensures that $\widetilde{B}_{+}$coincides with the quantity $B_{+}$of the theorem. We proceed in the same way with $F_{-}$. Summing up, we arrive at

$$
\begin{aligned}
\operatorname{Ent}_{\mu}\left(f^{2}\right) & \leq 4 B_{+} \int\left(F_{+}^{\prime}\right)^{2} d \nu+4 B_{-} \int\left(F_{-}^{\prime}\right)^{2} d \nu \\
& \leq 4 \max \left(B_{+}, B_{-}\right) \int\left(\left(F_{+}^{\prime}\right)^{2}+\left(F_{-}^{\prime}\right)^{2}\right) d \nu \\
& =4 \max \left(B_{+}, B_{-}\right) \int f^{\prime 2} d \nu .
\end{aligned}
$$

Indeed, $\left(F_{+}^{\prime}\right)^{2}+\left(F_{-}^{\prime}\right)^{2}=f^{\prime 2}$ at least on $\mathbb{R} \backslash\{m\}$. It follows that $C \leq$ $4 \max \left(B_{+}, B_{-}\right)$.

Next we give a lower bound on $C$ such that for every smooth non-negative $f$ on the line, $\operatorname{Ent}_{\mu}\left(f^{2}\right) \leq C \int f^{\prime 2} d \nu$. Let $f$ be a continuous function that vanishes on $(-\infty, m]$ and is smooth on $[m, \infty)$. By approximation, the latter inequality holds for such an $f$. Considering the variational expression (6) of $\operatorname{Ent}_{\mu}\left(f^{2}\right)$ and noting as before that the values of the test function $g$ outside the support of $f^{2}$ appear only in the constraint and have to be minimum $($ here $-\infty$ ) in order to approach the supremum, one writes 


$$
\begin{aligned}
\operatorname{Ent}_{\mu}\left(f^{2}\right) & =\sup \left\{\int_{(m, \infty)} f^{2} g d \mu ; \int_{(m, \infty)} e^{g} d \mu \leq 1\right\} \\
& \geq \sup \left\{\int_{(m, \infty)} f^{2} g d \mu ; \int_{(m, \infty)} e^{g} d \mu \leq 1 \text { and } g \geq 0\right\} .
\end{aligned}
$$

The latter supremum is non-trivial since the total mass of $\mu$ restricted to $(m, \infty)$ is at most $1 / 2$, so $\int_{(m, \infty)} e^{g} d \mu \leq 1$ can happen for many non-negative functions. From the log Sobolev inequality we deduce that for every smooth $f$ with $f(m)=0$ one has

$$
\sup \left\{\int_{(m, \infty)} f^{2} g d \mu ; \int_{(m, \infty)} e^{g} d \mu \leq 1 \text { and } g \geq 0\right\} \leq C \int_{(m, \infty)} f^{\prime 2} d \nu .
$$

Proposition 2 ensures that

$$
C \geq \sup _{x>m}\left[\sup \left\{\int_{(m, \infty)} \mathbf{1}_{[x, \infty)} g d \mu ; \int_{(m, \infty)} e^{g} d \mu \leq 1 \text { and } g \geq 0\right\}\right] \int_{m}^{x} \frac{1}{n} .
$$

Since $\mu((m, \infty)) \leq 1 / 2$, Lemma 6 applies, and shows that the latter is at least $b_{+}$. Doing the same on $(-\infty, m]$ we finally obtain $C \geq \max \left(b_{+}, b_{-}\right)$. The proof is therefore complete.

REMARK 7. It is not difficult to check that for any $0 \leq y \leq 1 / 2$,

$$
\log \left(1+\frac{e^{2}}{y}\right) \leq \frac{\log \left(1+2 e^{2}\right)}{\log (2)} \log \left(1+\frac{1}{2 y}\right) \leq 4 \log \left(1+\frac{1}{2 y}\right)
$$

Thus, $B_{+} \leq 4 b_{+}$and $B_{-} \leq 4 b_{-}$. It follows that the best logarithmic Sobolev constant $C$ satisfies $\max \left(\bar{b}_{-}, b_{+}\right) \leq C \leq 16 \max \left(b_{-}, b_{+}\right)$.

3. Sobolev inequalities. Let $p \in(1,2), \mu$ be a Borel probability measure on $\mathbb{R}$ and $d \nu(x)=n(x) d x$. In this section we show how the argument of the previous section may be adapted to the study of the optimal constant $C$ such that for every smooth $f: \mathbb{R} \rightarrow \mathbb{R}$ one has

$$
\int f^{2} d \mu-\left(\int|f|^{p} d \mu\right)^{2 / p} \leq C \int f^{\prime 2} d \nu
$$

The following lemma is a slight extension of Lemma 4.1 in [2].

Lemma 8. Let $p \in(1,2)$. Let $f: X \rightarrow \mathbb{R}$ be a square integrable function on a probability space $(X, Q)$. Then for all $a \in \mathbb{R}$ one has

$$
\int f^{2} d Q-\left(\int|f|^{p} d Q\right)^{2 / p} \leq \int(f-a)^{2} d Q-(p-1)\left(\int|f-a|^{p} d Q\right)^{2 / p}
$$


Proof. Let $g: X \rightarrow \mathbb{R}$ be a bounded function taking finitely many values. One studies $\varphi(t)=\left(\int|1+t g|^{p}\right)^{2 / p}$. For brevity we omit the measure $d Q$ in the notation. Clearly $\varphi(0)=1, \varphi^{\prime}(0)=2 \int g$ and

$$
\begin{aligned}
\frac{\varphi^{\prime \prime}(t)}{2}= & (2-p)\left(\int|1+t g|^{p}\right)^{2 / p-2}\left(\int g|1+t g|^{p-1} \operatorname{sign}(1+t g)\right)^{2} \\
& +(p-1)\left(\int|1+t g|^{p}\right)^{2 / p-1} \int g^{2}|1+t g|^{p-2} .
\end{aligned}
$$

The above differentiation is legitimate at least for all but a finite number of $t$ 's (where $1+t g$ vanishes). For such values, note that the first term in the second derivative is non-negative, while the second can be bounded from below by Hölder's inequality:

$$
\begin{aligned}
\int|g|^{p} & =\int|g|^{p}|1+t g|^{p(p-2) / 2} \cdot|1+t g|^{p(2-p) / 2} \\
& \leq\left(\int g^{2}|1+t g|^{p-2}\right)^{p / 2}\left(\int|1+t g|^{p}\right)^{(2-p) / 2} .
\end{aligned}
$$

Therefore $\varphi^{\prime \prime}(t) / 2 \geq(p-1)\left(\int|g|^{p}\right)^{2 / p}$. Since $\varphi$ is $\mathcal{C}^{1}$ (or convex), this is sufficient to deduce that $\varphi(t) \geq \varphi(0)+t \varphi^{\prime}(0)+t^{2}(p-1)\left(\int|g|^{p}\right)^{2 / p}$ for all $t$. Setting $a=1 / t$ and multiplying by $a^{2}$ one gets

$$
\left(\int|a+g|^{p}\right)^{2 / p} \geq a^{2}+2 a \int g+(p-1)\left(\int|g|^{p}\right)^{2 / p} .
$$

Subtracting this from the relation $\int(a+g)^{2}=a^{2}+2 a \int g+\int g^{2}$ gives the result for $f=a+g$. The general case follows by approximation.

Next we need a convenient representation of the left hand side in a Sobolev inequality as a supremum.

Lemma 9. Let $\varphi$ be a non-negative integrable function on a probability space $(X, P)$. Let $A>0$ and $a \in(0,1)$. Then

$$
\begin{aligned}
& \int \varphi d P-A\left(\int \varphi^{a} d P\right)^{1 / a} \\
& \quad=\sup \left\{\int \varphi g d P ; g<1 \text { and } \int(1-g)^{a /(a-1)} d P \leq A^{a /(a-1)}\right\} \\
& \quad \leq \sup \left\{\int \varphi g d P ; 0 \leq g<1 \text { and } \int(1-g)^{a /(a-1)} d P \leq 1+A^{a /(a-1)}\right\} .
\end{aligned}
$$

Proof. By a simple argument using Hölder's inequality, it is not difficult to see that

$$
\left(\int \varphi^{a} d P\right)^{1 / a}=\inf \left\{\int \varphi h d P ; h>0 \text { and } \int h^{a /(a-1)} d P \leq 1\right\} .
$$

Setting $g=1-A h$, we deduce from this fact that 


$$
\begin{aligned}
\int \varphi d P & -A\left(\int \varphi^{a} d P\right)^{1 / a} \\
& =\sup \left\{\int \varphi(1-A h) d P ; h>0 \text { and } \int h^{a /(a-1)} d P \leq 1\right\} \\
& =\sup \left\{\int \varphi g d P ; g<1 \text { and } \int(1-g)^{a /(a-1)} d P \leq A^{a /(a-1)}\right\} \\
& \leq \sup \left\{\int \varphi g \mathbf{1}_{g \geq 0} d P ; g<1 \text { and } \int(1-g)^{a /(a-1)} d P \leq A^{a /(a-1)}\right\} \\
& \leq \sup \left\{\int \varphi g d P ; 0 \leq g<1 \text { and } \int(1-g)^{a /(a-1)} \leq 1+A^{a /(a-1)}\right\} .
\end{aligned}
$$

We have used the following simple estimate:

$$
\begin{aligned}
\int\left(1-g \mathbf{1}_{g \geq 0}\right)^{a /(a-1)} d P & =\int(1-g)^{a /(a-1)} 1_{g \geq 0} d P+P\{g<0\} \\
& \leq \int(1-g)^{a /(a-1)} d P+1 .
\end{aligned}
$$

We shall need the following analogue of Lemma 6. Its proof is very similar and we omit it.

LEMma 10. Let $a \in(0,1)$. Let $Q$ be a finite measure on a space $X$ and let $K>Q(X)$. Let $A \subset X$ be measurable with $Q(A)>0$. Then

$$
\begin{aligned}
\sup \left\{\int_{X} \mathbf{1}_{A} g d Q ; 0 \leq g<1\right. \text { and } & \left.\int_{X}(1-g)^{a /(a-1)} d Q \leq K\right\} \\
& =Q(A)\left(1-\left(1+\frac{K-Q(X)}{Q(A)}\right)^{(a-1) / a}\right) .
\end{aligned}
$$

We are now in a position to state our characterization of measures which satisfy the inequality (7).

TheOREM 11. Let $p \in(1,2)$ and let $\mu, \nu$ be Borel measures on $\mathbb{R}$ with $\mu(\mathbb{R})=1$ and $d \nu(x)=n(x) d x$. Let $m$ be a median of $\mu$. Let $C$ be the optimal constant such that for every smooth $f: \mathbb{R} \rightarrow \mathbb{R}$ one has

$$
\int f^{2} d \mu-\left(\int|f|^{p} d \mu\right)^{2 / p} \leq C \int f^{\prime 2} d \nu
$$

Then $\max \left(b_{-}(p), b_{+}(p)\right) \leq C \leq 4 \max \left(B_{-}(p), B_{+}(p)\right)$ where

$$
\begin{aligned}
b_{+}(p) & =\sup _{x>m} \mu([x, \infty))\left(1-\left(1+\frac{1}{2 \mu([x, \infty))}\right)^{(p-2) / p}\right) \int_{m}^{x} \frac{1}{n} \\
B_{+}(p) & =\sup _{x>m} \mu([x, \infty))\left(1-\left(1+\frac{(p-1)^{p /(p-2)}}{\mu([x, \infty))}\right)^{(p-2) / p}\right) \int_{m}^{x} \frac{1}{n},
\end{aligned}
$$




$$
\begin{aligned}
& b_{-}(p)=\sup _{x<m} \mu((-\infty, x])\left(1-\left(1+\frac{1}{2 \mu((-\infty, x])}\right)^{(p-2) / p}\right) \int_{x}^{m} \frac{1}{n} \\
& B_{-}(p)=\sup _{x<m} \mu((-\infty, x])\left(1-\left(1+\frac{(p-1)^{p /(p-2)}}{\mu((-\infty, x])}\right)^{(p-2) / p}\right) \int_{x}^{m} \frac{1}{n} .
\end{aligned}
$$

Proof. The proof is similar to the one of Theorem 3. Just use Lemmas 8, 9 (with $A=p-1$ and $a=p / 2$ ) and 10 instead of Lemmas 4, 5 and 6 .

REMARK 12. One can check that for any $0 \leq y \leq 1 / 2$ and any $p \in(1,2)$,

$$
\frac{1-\left(1+\frac{(p-1)^{p /(p-2)}}{y}\right)^{(p-2) / p}}{1-\left(1+\frac{1}{2 y}\right)^{(p-2) / p}} \leq \frac{1-\left(1+2(p-1)^{p /(p-2)}\right)^{(p-2) / p}}{1-2^{(p-2) / p}} \leq 5 .
$$

Thus, $B_{+}(p) \leq 5 b_{+}(p)$ and $B_{-}(p) \leq 5 b_{-}(p)$. It follows that the best constant $C$ in (8) satisfies $\max \left(b_{-}(p), b_{+}(p)\right) \leq C \leq 20 \max \left(b_{-}(p), b_{+}(p)\right)$.

We now explain how the latter theorem gives a characterization of those measures which satisfy the Latała-Oleszkiewicz inequality.

TheOREm 13. Let $r \in(1,2)$. Let $\mu, \nu$ be Borel measures on $\mathbb{R}$ with $\mu(\mathbb{R})=1$ and $d \nu(x)=n(x) d x$. Let $m$ be a median of $\mu$. Let $C$ be the optimal constant such that for any smooth $f: \mathbb{R} \rightarrow \mathbb{R}$ one has

$$
\sup _{p \in(1,2)} \frac{\int f^{2} d \mu-\left(\int|f|^{p} d \mu\right)^{2 / p}}{(2-p)^{2(1-1 / r)}} \leq C \int f^{\prime 2} d \nu .
$$

\section{Then}

$$
\frac{1}{3} \max \left(c_{-}(r), c_{+}(r)\right) \leq C \leq 4 \max \left(C_{-}(r), C_{+}(r)\right) \leq 17 \max \left(c_{-}(r), c_{+}(r)\right)
$$

where

$$
\begin{aligned}
& c_{+}(r)=\sup _{x>m} \mu([x, \infty))\left(\log \left(1+\frac{1}{2 \mu([x, \infty))}\right)\right)^{2(1-1 / r)} \int_{m}^{x} \frac{1}{n}, \\
& C_{+}(r)=\sup _{x>m} \mu([x, \infty))\left(\log \left(1+\frac{8}{\mu([x, \infty))}\right)\right)^{2(1-1 / r)} \int_{m}^{x} \frac{1}{n}, \\
& c_{-}(r)=\sup _{x<m} \mu((-\infty, x])\left(\log \left(1+\frac{1}{2 \mu((-\infty, x])}\right)\right)^{2(1-1 / r)} \int_{x}^{m} \frac{1}{n}, \\
& C_{-}(r)=\sup _{x<m} \mu((-\infty, x])\left(\log \left(1+\frac{8}{\mu((-\infty, x])}\right)\right)^{2(1-1 / r)} \int_{x}^{m} \frac{1}{n} .
\end{aligned}
$$


Proof. Using Theorem 11 for any $p \in(1,2)$, one gets

$$
\sup _{p \in(1,2)} \frac{\max \left(b_{-}(p), b_{+}(p)\right)}{(2-p)^{2(1-1 / r)}} \leq C \leq 4 \sup _{p \in(1,2)} \frac{\max \left(B_{-}(p), B_{+}(p)\right)}{(2-p)^{2(1-1 / r)}} .
$$

Therefore, it follows from Lemma 14 below applied with $\tau=2(1-1 / r)$ that $\frac{1}{3} \max \left(c_{-}(r), c_{+}(r)\right) \leq C \leq 4 \max \left(C_{-}(r), C_{+}(r)\right)$. To complete the proof, one proceeds as in Remarks 7, 12.

Lemma 14. For any $0<y \leq 1 / 2$ and any $\tau \in[0,1]$,

$$
\sup _{p \in(1,2)} \frac{1-\left(1+\frac{(p-1)^{p /(p-2)}}{y}\right)^{(p-2) / p}}{(2-p)^{\tau}} \leq\left[\log \left(1+\frac{8}{y}\right)\right]^{\tau}
$$

and

$$
\sup _{p \in(1,2)} \frac{1-\left(1+\frac{1}{2 y}\right)^{(p-2) / p}}{(2-p)^{\tau}} \geq \frac{1}{3}\left[\log \left(1+\frac{1}{2 y}\right)\right]^{\tau} .
$$

Proof. The term $A(p)=(p-1)^{p /(p-2)}$ is just a little nuisance. As $A(p)$ is non-increasing in $p, A(p) \leq 8$ for $p \in[3 / 2,2)$. Thus,

$$
\begin{aligned}
& \sup _{p \in(1,2)} \frac{1-\left(1+(p-1)^{p /(p-2)} / y\right)^{(p-2) / p}}{(2-p)^{\tau}} \\
& \leq \max \left[\sup _{p \in(1,3 / 2)} \frac{1}{(2-p)^{\tau}}, \sup _{p \in[3 / 2,2)} \frac{1-(1+8 / y)^{(p-2) / p}}{(2-p)^{\tau}}\right] .
\end{aligned}
$$

Set $b=(2-p) / p$ and note that $2-p=2 b /(b+1)$. For $p \in[3 / 2,2)$, one has $b \in(0,1 / 3]$ and therefore $(3 / 2) b \leq 2 b /(b+1) \leq 2 b$. Changing variable and using the lower bound in the latter inequality, we estimate from above the second supremum by $\sup _{b \in(0,1 / 3]}\left(1-X^{-b}\right) / b^{\tau}$ where $X=1+8 / y \in[17, \infty)$. Next, put $c=b \log X$. Then

$$
\sup _{b \in(0,1 / 3]} \frac{1-X^{-b}}{b^{\tau}}=(\log X)^{\tau} \sup _{c \in\left(0, \frac{1}{3} \log X\right]} \frac{1-e^{-c}}{c^{\tau}} \leq(\log X)^{\tau},
$$

because $\sup _{c>0}\left(1-e^{-c}\right) / c^{\tau} \leq 1$. Finally,

$$
\sup _{p \in(1,3 / 2)}(2-p)^{-\tau}=2^{\tau} \leq(\log X)^{\tau}
$$

This proves the first part of the lemma.

For the second part, we proceed as before with $b \in(0,1)$ and set $X=$ $1+1 / 2 y \in[2, \infty)$. It follows that 


$$
\begin{aligned}
& \sup _{p \in(1,2)} \frac{1-(1+1 / 2 y)^{(p-2) / p}}{(2-p)^{\tau}} \geq \frac{1}{2^{\tau}}(\log X)^{\tau} \sup _{c \in(0, \log 2)} \frac{1-e^{-c}}{c^{\tau}} \\
& \geq \frac{1}{2(2 \log 2)^{\tau}}(\log X)^{\tau},
\end{aligned}
$$

where we have taken $c=\log 2$. Finally

$$
\frac{1}{2(2 \log 2)^{\tau}} \geq \frac{1}{4 \log 2} \geq \frac{1}{3}
$$

Next, we give examples of applications of Theorem 13.

Proposition 15. Let $d \mu(x)=e^{-\Phi(x)} d x$ be a probability measure, where $\Phi$ is a continuous function on $\mathbb{R}$ satisfying:

(i) there exists $A>0$ such that for $|x|>A, \Phi$ is $\mathcal{C}^{2}$ and $\operatorname{sign}(x) \Phi^{\prime}(x)>0$,

(ii) $\lim _{|x| \rightarrow \infty} \Phi^{\prime \prime}(x) / \Phi^{\prime}(x)^{2}=0$,

(iii) there exists $r \in(1,2)$ such that

$$
\limsup _{|x| \rightarrow \infty} \frac{\left[\Phi(x)+\log \Phi^{\prime}(x)\right]^{2(1-1 / r)}}{\Phi^{\prime}(x)^{2}}<\infty .
$$

Then there exists $C \geq 0$ such that $\mu$ satisfies the Latała-Oleszkiewicz inequality (9) with the corresponding $r$ (and $\nu=\mu$ on the right hand side).

Proof. Let $m$ be a median of $\mu$. Under our hypotheses, when $x$ tends to $\infty$, one has (see e.g. [1, Chapter 6])

$$
\int_{m}^{x} e^{\Phi(t)} d t \sim \frac{e^{\Phi(x)}}{\Phi^{\prime}(x)}, \quad \int_{x}^{\infty} e^{-\Phi(t)} d t \sim \frac{e^{-\Phi(x)}}{\Phi^{\prime}(x)} .
$$

Thus, for any $x \geq m$,

$$
\mu([x, \infty))\left(\log \left(1+\frac{8}{\mu([x, \infty))}\right)\right)^{2(1-1 / r)} \int_{m}^{x} \frac{1}{n} \sim \frac{\left[\Phi(x)+\log \Phi^{\prime}(x)\right]^{2(1-1 / r)}}{\Phi^{\prime}(x)^{2}} .
$$

By hypothesis (iii), this quantity is bounded on $\left[A^{\prime}, \infty\right)$ for some $A^{\prime}>0$. As it is continuous on $\left[m, A^{\prime}\right]$, it is bounded on $(m, \infty)$. Thus, $C_{+}(r)<\infty$, and similarly for $C_{-}(r)$. We conclude by applying Theorem 13 .

As a direct application, we recover that the measures

$$
d \nu_{r}(t)=\exp \left(-|t|^{r}\right) \frac{d t}{2 \Gamma(1+1 / r)}, \quad r \in(1,2),
$$

satisfy the Latała-Oleszkiewicz inequality (3) with the corresponding $r$, and a constant $C$ that may depend on $r$. To recover the full result of [11], namely that $C$ can be chosen independent of $r \in(1,2)$, one can combine Theorem 13 with the classical estimates on $\int_{0}^{x} \exp \left(t^{r}\right) d r$ and $\int_{x}^{\infty} \exp \left(-t^{r}\right) d r$. This is very easy and we omit the details of the argument. The criterion allows one to 
deal with more general potentials, which are not bounded perturbations of the latter. For example, we find that the probability measures

$$
\begin{aligned}
d \tau_{r}(x)=\exp \left(-|x|^{r}-|x|^{r-1} \cos (x)\right) \frac{d x}{Z_{r}}, & r \in(1,2), \\
d \gamma_{r, a}(x)=x^{a} \exp \left(-x^{r}\right) \mathbf{1}_{x>0} \frac{r d x}{\Gamma((a+1) / r)}, & r \in(1,2), a>-1,
\end{aligned}
$$

also satisfy the Latała-Oleszkiewicz inequality (3) with the corresponding $r$. These facts, or more generally Theorem 13, can be combined with the following result of these authors in order to establish dimension free concentration inequalities with decay $\exp \left(-t^{r}\right)$ for product measures:

Theorem 16 ([11]). Let $r \in[1,2]$ and $C>0$. Let $\mu$ be a probability measure on $\mathbb{R}^{k}$. Assume that for any smooth $f: \mathbb{R}^{k} \rightarrow \mathbb{R}$ and any $p \in[1,2)$, one has

$$
\int f^{2} d \mu-\left(\int|f|^{p} d \mu\right)^{2 / p} \leq C(2-p)^{2(1-1 / r)} \int|\nabla f|^{2} d \mu
$$

Then for any integer $n \geq 1$ and any $h: \mathbb{R}^{n k} \rightarrow \mathbb{R}$ with $\|h\|_{\text {Lip }} \leq 1$ (with respect to the Euclidean norm on $\left.\mathbb{R}^{n k}\right)$, one has $\int|h| d \mu^{n}<\infty$ and

$$
\begin{gathered}
\mu^{n}\left(\left\{x ; h(x)-\int h d \mu^{n} \geq t \sqrt{C}\right\}\right) \leq e^{-t^{2} / 3}, \quad t \in[0,1], \\
\mu^{n}\left(\left\{x ; h(x)-\int h d \mu^{n} \geq t \sqrt{C}\right\}\right) \leq e^{-t^{r} / 3}, \quad t \geq 1 .
\end{gathered}
$$

Following this route Latała and Oleszkiewicz proved dimension free concentration inequalities for $\nu_{r}^{n}$. For these measures, a slightly stronger statement was proved earlier by Talagrand, via inf-convolution inequalities and transport, which holds for even log-concave densities on the line [18, Proposition 2.7.4]. The inf-convolution approach seems a bit more general than the one based on Sobolev inequalities. However, there exists no simple criterion so far that would allow one to decide whether a measure on $\mathbb{R}$ satisfies an inf-convolution inequality. On the other hand, Theorems 13 and 16 form a convenient tool to obtain new concentration inequalities for wide families of measures, including e.g. $\tau_{r}^{n}, \gamma_{r, a}^{n}$.

4. Final remarks. We conclude with some remarks and extensions.

1) As mentioned in [4], a Sobolev type inequality holds with right hand side $C \int f^{\prime 2}(x) d \nu(x)$ if and only if it holds with $C \int f^{\prime 2}(x) n(x) d x$ where $n$ is the absolutely continuous part of $\nu$. So our results extend to not necessarily absolutely continuous $\nu$ on $\mathbb{R}$. Only $n$ enters the criterion.

2) The method presented here can actually be extended to deal with more general concentration regimes, between exponential and Gaussian, but not necessarily $\exp \left(-t^{r}\right)$. The details will be given in a paper in preparation. 
3) Sobolev inequalities for $p>2$. Our method can easily be adapted to the Sobolev inequality (4) for $p>2$, for measures on $\mathbb{R}$. It would give good estimates for fixed $p$ or even when $p \rightarrow 2$. However, the result of Lemma 8 (reversed for $p>2$ ) introduces a $p-1$ in front of the $L_{p}$-norm. This term spoils our estimates as $p \rightarrow \infty$.

4) Other splittings. In our arguments we split the measures on $\mathbb{R}$ into two measures on the half-lines starting at the median $m$ of $\mu$. The argument works if one makes the cut at some other point $a$. The upper estimate on the constant in Sobolev type inequalities would be similar, with $m$ replaced by $a$. In the lower bound, the mass of $\mu$ on the left and on the right of $a$ would appear. If not the most convenient in practice, the choice of the median seems to be the best.

5) Discrete case. The argument of Sections 2 and 3 can easily be adapted to measures on $\mathbb{Z}$. If $\mu$ is a probability measure and $\nu$ a positive measure, both on $\mathbb{Z}$, then the logarithmic Sobolev inequality reads

$$
\operatorname{Ent}_{\mu}\left(f^{2}\right) \leq C \sum_{x \in \mathbb{Z}} \nu(x)(f(x+1)-f(x))^{2}
$$

for every $f: \mathbb{Z} \rightarrow \mathbb{R}$ null except at a finite number of points, with $\operatorname{Ent}_{\mu}\left(f^{2}\right)=$ $\mu\left(f^{2} \log f^{2}\right)-\mu\left(f^{2}\right) \log \mu\left(f^{2}\right)$. In the same way, one can state a Sobolev inequality in the discrete setting.

Exactly as in the proof of Theorem 3, one can prove that the best constant $C$ in (10) satisfies $\max \left(b_{-}, b_{+}\right) \leq C \leq 4 \max \left(B_{-}, B_{+}\right)$with

$$
B_{+}=\sup _{x>m}\left(\sum_{y \geq x} \mu(y)\right) \log \left(1+\frac{e^{2}}{\sum_{y \geq x} \mu(y)}\right) \sum_{y=m+1}^{x} \frac{1}{\nu(y)}
$$

and similarly for $b_{-}, b_{+}$and $B_{-}$. The same remark applies to Section 3 about Sobolev inequalities and inequality (9).

6) Inequalities in $\mathbb{R}^{n}$. One can give a criterion for functional inequalities on $\mathbb{R}^{n}$ (it is however difficult to exploit). We illustrate this on the Poincaré inequality. Let $f: \mathbb{R}^{n} \rightarrow \mathbb{R}$ be smooth. Then $\operatorname{Var}_{\mu}(f) \leq \int(f-m)^{2} d \mu$ where $m$ is a median of the law of $f$ under $\mu$. Set $F_{+}=(f-m) \mathbf{1}_{\{f>m\}}$ and $F_{-}=(f-m) \mathbf{1}_{\{f<m\}}$. One has $\int(f-m)^{2} d \mu=\int F_{-}^{2} d \mu+\int F_{+}^{2} d \mu$. Now, fix $\Omega=\{x ; f(x)>m\}$. A result of Maz'ya [13] asserts that the best constant $\alpha$ in $\int_{\Omega} F^{2} d \mu \leq \alpha \int_{\Omega}|\nabla F|^{2} d \nu$ for smooth $F$ vanishing on $\partial \Omega$ (and at infinity, but we can get rid of this by approximation since $\mu$ is finite) is, up to universal constants, equal to the best $\beta$ such that for every open set $A \subset \Omega$, one has $\beta \operatorname{Cap}_{\nu}(A, \Omega) \geq \mu(A)$ where

$$
\operatorname{Cap}_{\nu}(A, \Omega)=\inf \left\{\int_{\Omega}|\nabla f|^{2} d \nu ; \mathbf{1}_{A} \leq f \leq \mathbf{1}_{\Omega}\right\}
$$


is the capacity of $A$ in $\Omega$ under $\mu$. The idea of his argument is mainly a decomposition of functions into level sets. In our case we have no idea of the shape of $\Omega=\{x ; f(x)>m\}$. So the relevant notion of capacity of a set $A$ such that $\mu(A)<1 / 2$ seems to be

$$
\operatorname{Cap}_{\nu}(A, \mu)=\inf \left\{\operatorname{Cap}_{\nu}(A, \Omega) ; \Omega \supset A \text { and } \mu(\Omega)=1 / 2\right\} .
$$

Let $\beta$ be the smallest number so that $\beta \operatorname{Cap}_{\nu}(A, \mu) \geq \mu(A)$ whenever $\mu(A) \leq$ $1 / 2$. Then, by Maz'ya's result, $\operatorname{Var}_{\mu}(f) \leq k \beta \int|\nabla f|^{2} d \nu$ for some universal constant $k$.

On the other hand, if $\int f^{2} d \mu-\left(\int f d \mu\right)^{2} \leq C \int|\nabla f|^{2} d \nu, \mu(\Omega)=1 / 2$ and $\mathbf{1}_{A} \leq f \leq \mathbf{1}_{\Omega}$, then

$$
\left(\int f d \mu\right)^{2}=\left(\int f \mathbf{1}_{\Omega} d \mu\right)^{2} \leq \frac{1}{2} \int f^{2} .
$$

So, $C \int|\nabla f|^{2} d \nu \geq \frac{1}{2} \int f^{2} \geq \frac{1}{2} \mu(A)$ and $C \operatorname{Cap}_{\nu}(A, \mu) \geq \frac{1}{2} \mu(A)$. It follows that $\frac{1}{2} \beta \leq C \leq k \beta$. We have thus obtained a characterization of those measures which satisfy a Poincaré inequality on $\mathbb{R}^{n}$.

Similarly the logarithmic Sobolev constant is equal, up to universal constants, to the best constant $\widetilde{\beta}$ in $\widetilde{\beta} \operatorname{Cap}_{\nu}(A, \mu) \geq \mu(A) \log (1 / \mu(A))$.

Finally, we show the link with dimension 1 . An intermediate step was to deal with the inequality $\int_{m}^{\infty} f^{2} d \mu \leq C \int_{m}^{\infty} f^{\prime 2} d \nu$ for $f(m)=0$. Such an inequality is made tighter by replacing $f(x)$ by $\int_{m}^{x}\left|f^{\prime}\right|$. So one reduces to increasing functions with level sets $(x, \infty) \subset(m, \infty)$. And the capacity $\operatorname{Cap}_{\nu}((x, \infty),(m, \infty))$ is easily seen to be $\left(\int_{m}^{x} 1 / n\right)^{-1}$ where $d \nu(x)=n(x) d x$. Indeed, if $f$ is a smooth function such that $\mathbf{1}_{(x, \infty)} \leq f \leq \mathbf{1}_{(m, \infty)}, f(m)=0$ and $f(x)=1$, then by the Cauchy-Schwarz inequality,

$$
\int_{m}^{\infty} f^{\prime 2} n=\int_{m}^{\infty} f^{\prime 2} n \geq \frac{\left(\int_{m}^{\infty} f^{\prime}\right)^{2}}{\int_{m}^{x} \frac{1}{n}}=\frac{1}{\int_{m}^{x} \frac{1}{n}} .
$$

This inequality is tight for appropriate choices of $f$, and the result follows.

Acknowledgements. We thank Krzysztof Oleszkiewicz for useful suggestions.

\section{References}

[1] C. Ané, S. Blachère, D. Chafaï, P. Fougères, I. Gentil, F. Malrieu, C. Roberto et G. Scheffer, Sur les inégalités de Sobolev logarithmiques, Panoramas et Synthèses 10, Soc. Math. France, Paris, 2000.

[2] D. Bakry, L'hypercontractivité et son utilisation en théorie des semigroupes, in: Lectures on Probability Theory (Saint-Flour, 1992), Lecture Notes in Math. 1581, Springer, Berlin, 1994, 1-114.

[3] W. Beckner, A generalized Poincaré inequality for Gaussian measures, Proc. Amer. Math. Soc. 105 (1989), 397-400. 
[4] S. G. Bobkov and F. Götze, Exponential integrability and transportation cost related to logarithmic Sobolev inequalities, J. Funct. Anal. 163 (1999), 1-28.

[5] M. F. Chen, Variational formulas of Poincaré type inequalities for birth and death processes, preprint, 2002.

[6] -, Variational formulas of Poincaré-type inequalities in Banach spaces of functions on the line, Acta Math. Sin. (Engl Ser.) 18 (2002), 417-436.

[7] L. Gross, Logarithmic Sobolev inequalities, Amer. J. Math. 97 (1975), 1061-1083.

[8] A. Guionnet and B. Zegarliński, Lectures on logarithmic Sobolev inequalities, in: Séminaire de Probabilité XXXVI, Lecture Notes in Math. 1801, Springer, Berlin, 2003, 1-134.

[9] G. H. Hardy, J. E. Littlewood and G. Pólya, Inequalities, Cambridge Math. Library, Cambridge Univ. Press, Cambridge, 1988 (reprint of the 1952 edition).

[10] B. Helffer, Semiclassical Analysis, Witten Laplacians, and Statistical Mechanics, Ser. Partial Differential Equations Appl. 1, World Sci., River Edge, NJ, 2002.

[11] R. Latała and K. Oleszkiewicz, Between Sobolev and Poincaré, in: Geometric Aspects of Functional Analysis, Lecture Notes in Math. 1745, Springer, Berlin, 2000, $147-168$.

[12] M. Ledoux, The Concentration of Measure Phenomenon, Math. Surveys Monographs 89, Amer. Math. Soc., Providence, RI, 2001.

[13] V. G. Maz'ya, Sobolev Spaces, Springer Ser. Soviet Math., Springer, Berlin, 1985 (translated from the Russian by T. O. Shaposhnikova).

[14] L. Miclo, An example of application of discrete Hardy's inequalities, Markov Process. Related Fields 5 (1999), 319-330.

[15] B. Muckenhoupt, Hardy inequalities with weights, Studia Math. 44 (1972), 31-38.

[16] O. S. Rothaus, Analytic inequalities, isoperimetric inequalities and logarithmic Sobolev inequalities, J. Funct. Anal. 64 (1985), 296-313.

[17] G. Royer, Une initiation aux inégalités de Sobolev logarithmiques, Cours Spécialisés 5, Soc. Math. France, Paris, 1999.

[18] M. Talagrand, Concentration of measure and isoperimetric inequalities in product spaces, Inst. Hautes Études Sci. Publ. Math. 81 (1995), 73-205.

[19] G. Talenti, Osservazioni sopra una classe di disuguaglianze, Rend. Sem. Mat. Fis. Milano 39 (1969), 171-185.

[20] G. Tomaselli, A class of inequalities, Boll. Un. Mat. Ital. (4) 2 (1969), 622-631.

[21] A. Zygmund, Trigonometric Series, Vols. I, II, 2nd ed., Cambridge Univ. Press, New York, 1959.

CNRS, Laboratoire d'Analyse et Mathématiques Appliquées, UMR 8050

Universités de Marne-la-Vallée et de Paris 12-Val-de-Marne

Boulevard Descartes, Cité Descartes, Champs sur Marne

77454 Marne-la-Vallée Cedex 2, France

E-mail: barthe@math.univ-mlv.fr

roberto@math.univ-mlv.fr

Received February 3, 2003

Revised version June 26, 2003 